\title{
Cost-effectiveness of cerebrospinal biomarkers for the diagnosis of Alzheimer's disease
}

\author{
Spencer A. W. Lee ${ }^{1,2}$, Luciano A. Sposato ${ }^{3,4,5}$, Vladimir Hachinski ${ }^{3,6}$ and Lauren E. Cipriano ${ }^{1,6^{*}}$ (D)
}

\begin{abstract}
Background: Accurate and timely diagnosis of Alzheimer's disease (AD) is important for prompt initiation of treatment in patients with $A D$ and to avoid inappropriate treatment of patients with false-positive diagnoses.

Methods: Using a Markov model, we estimated the lifetime costs and quality-adjusted life-years (QALYS) of cerebrospinal fluid biomarker analysis in a cohort of patients referred to a neurologist or memory clinic with suspected AD who remained without a definitive diagnosis of $A D$ or another condition after neuroimaging. Parametric values were estimated from previous health economic models and the medical literature. Extensive deterministic and probabilistic sensitivity analyses were performed to evaluate the robustness of the results.
\end{abstract}

Results: At a 12.7\% pretest probability of AD, biomarker analysis after normal neuroimaging findings has an incremental cost-effectiveness ratio (ICER) of $\$ 11,032$ per QALY gained. Results were sensitive to the pretest prevalence of $A D$, and the ICER increased to over $\$ 50,000$ per QALY when the prevalence of AD fell below 9\%. Results were also sensitive to patient age (biomarkers are less cost-effective in older cohorts), treatment uptake and adherence, biomarker test characteristics, and the degree to which patients with suspected AD who do not have AD benefit from AD treatment when they are falsely diagnosed.

Conclusions: The cost-effectiveness of biomarker analysis depends critically on the prevalence of AD in the tested population. In general practice, where the prevalence of $A D$ after clinical assessment and normal neuroimaging findings may be low, biomarker analysis is unlikely to be cost-effective at a willingness-to-pay threshold of $\$ 50,000$ per QALY gained. However, when at least 1 in 11 patients has AD after normal neuroimaging findings, biomarker analysis is likely cost-effective. Specifically, for patients referred to memory clinics with memory impairment who do not present neuroimaging evidence of medial temporal lobe atrophy, pretest prevalence of AD may exceed 15\%. Biomarker analysis is a potentially cost-saving diagnostic method and should be considered for adoption in high-prevalence centers.

Keywords: Alzheimer's disease, Cost-effectiveness analysis, Cerebrospinal fluid biomarkers, Neuroimaging

\section{Background}

Alzheimer's disease (AD) is a progressive neurodegenerative disorder currently affecting an estimated 36 million people globally, with prevalence predicted to double in the next 10 years [1-3]. In the United States alone, with 5.2 million patients with $\mathrm{AD}[4]$, total direct costs in 2014 were estimated to be $\$ 214$ billion, with another

\footnotetext{
* Correspondence: Icipriano@ivey.uwo.ca

${ }^{1}$ Ivey Business School, Western University, 1255 Western Road, London, ON N6G 0N1, Canada

${ }^{6}$ Department of Biostatistics and Epidemiology, Schulich School of Medicine and Dentistry, Western University, London, ON N6A 5C1, Canada

Full list of author information is available at the end of the article
}

$\$ 220$ billion in unpaid care [1]. Accurate and timely diagnosis of $\mathrm{AD}$ is important to initiate treatment promptly and to avoid inappropriate therapeutic interventions in patients with false-positive diagnoses [5]. Even though current treatments (acetylcholinesterase inhibitors and memantine) do not reverse the underlying neurological damage, $\mathrm{AD}$ treatments can delay cognitive and functional decline and improve overall quality of life $[6,7]$. Several studies have found AD treatments to be cost-effective in mild to moderate $\mathrm{AD}$ and moderate to severe $\mathrm{AD}[8-11]$. 
Clinical diagnosis of AD has a relatively low and highly uncertain diagnostic accuracy $[12,13]$. To aid in diagnosis, neuroimaging by computed tomography $(\mathrm{CT})$ or magnetic resonance imaging (MRI) is typically performed, both to rule out non-AD causes of cognitive impairment, such as meningioma and subdural hematoma, and to evaluate structural indicators of $\mathrm{AD}$, including medial temporal lobe (MTL) atrophy [14]. Still, these neuroimaging techniques do not provide the desired level of accuracy to confidently diagnose $\mathrm{AD}$ in a considerable proportion of patients. Single-photon emission computed tomography (SPECT), ${ }^{18}$ F-fluorodeoxyglucose positron emission tomography (PET), and amyloid PET are effective at ruling out a diagnosis of neurodegenerative disease and amyloid- $\beta(A \beta)$ deposition in the brain, but the results are complex, difficult to interpret, and have low to moderate positive predictive value, especially in older patients because brain $A \beta$ deposition increases with age [14-16].

Cerebrospinal fluid (CSF) biomarkers have demonstrated relatively high diagnostic accuracy even for prodromal $\mathrm{AD}$ in patients with mild cognitive impairment (MCI) $[14,15]$ and so may provide additional diagnostic insight. However, CSF collection involves a lumbar puncture, which has an associated cost and causes patient discomfort.

Previous cost-effectiveness analyses of AD diagnostic technologies present conflicting findings potentially attributable to differences in the clinical setting of the diagnosis being considered $[17,18]$. In two studies performed in the early 2000s, researchers found the addition of SPECT and PET to clinical assessment was not cost-effective $[19,20]$. Authors of a recent costeffectiveness analysis compared clinical assessment plus florbetapir-PET with clinical assessment alone and found the addition of florbetapir-PET to be cost-effective from the perspective of the Spanish National Health System [21]. However, they did not compare PET with a standard diagnostic regimen including CT or MRI analysis. Researchers in a cost minimization study, also performed from the perspective of the Spanish National Health System, suggested that the use of CSF biomarkers may reduce AD-related health care costs [22]. However, that study did not account for the discomfort and risks of undergoing lumbar puncture or improvements in quality of life for patients accurately diagnosed with $\mathrm{AD}$. In the present study, we evaluated the cost-effectiveness of performing CSF biomarker analysis in a cohort of patients with suspected dementia who were referred to a neurologist or memory clinic and who remained without a definitive diagnosis after neuroimaging.

\section{Methods}

We developed a Markov model to evaluate the lifetime costs and benefits of performing CSF biomarker analysis in patients referred to a neurologist or memory clinic with suspected dementia who, after evaluation by neuroimaging, do not have a definitive diagnosis of $\mathrm{AD}$ or another cause of dementia (Fig. 1a). In 1-month time steps, the model followed the diagnosis and health state progression of a hypothetical cohort of patients. We used standard health economic methods by taking a societal perspective, considering costs and benefits over a lifetime horizon, discounting costs and benefits at 3\% annually, and performing both probabilistic and deterministic sensitivity analysis to evaluate the robustness of our findings [23]. For determining cost-effectiveness, we used the commonly applied thresholds of $\$ 50,000$ and $\$ 100,000$ per quality-adjusted life-year (QALY) gained [24]. We implemented the model in Microsoft Excel 2013 using Visual Basic for Applications (Microsoft Corp., Seattle, WA, USA).

\section{Model overview}

A schematic of the model is presented in Fig. 1. We considered two diagnostic strategies: biomarker analysis and do nothing. Patients were divided into four groups on the basis of their true health state and diagnosed health state: true-positive, false-negative, false-positive, and true-negative (Fig. 1b). Similar to previously published model-based analyses of AD [19], individuals who had $\mathrm{AD}$ were divided into 12 health states on the basis of the severity of their disease, whether or not they were on treatment, and their location (Fig. 1c). In the base case analysis, we assumed that patients who did not have $\mathrm{AD}$ had another disease causing stable MCI, so individuals who did not have AD were divided into four health states on the basis of whether they were on AD treatment (because of false diagnosis) and their type of residence (Fig. 1d). We performed structural sensitivity analysis exploring alternative assumptions for the natural history for the non-AD patients, including modeling it as a stable, moderate cognitive impairment and as a progressive cognitive impairment with transition rates similar to AD.

In each month, patients could die or transition from one health state to another. We estimated the rate of transition between disease states, the influence of treatment on those transitions, as well as costs and utilities associated with each health state from the medical literature (Table 1). When multiple sources were available to inform parameters, we selected studies that were more generalizable to the modeled population (i.e., large, U.S.-based cohorts) and those using more recent datasets. When the literature reported conflicting evidence or wide uncertainty, we selected a central value for the base case and performed extensive sensitivity analysis over the entire range of values reported in the literature. We validated model outcomes by replicating 

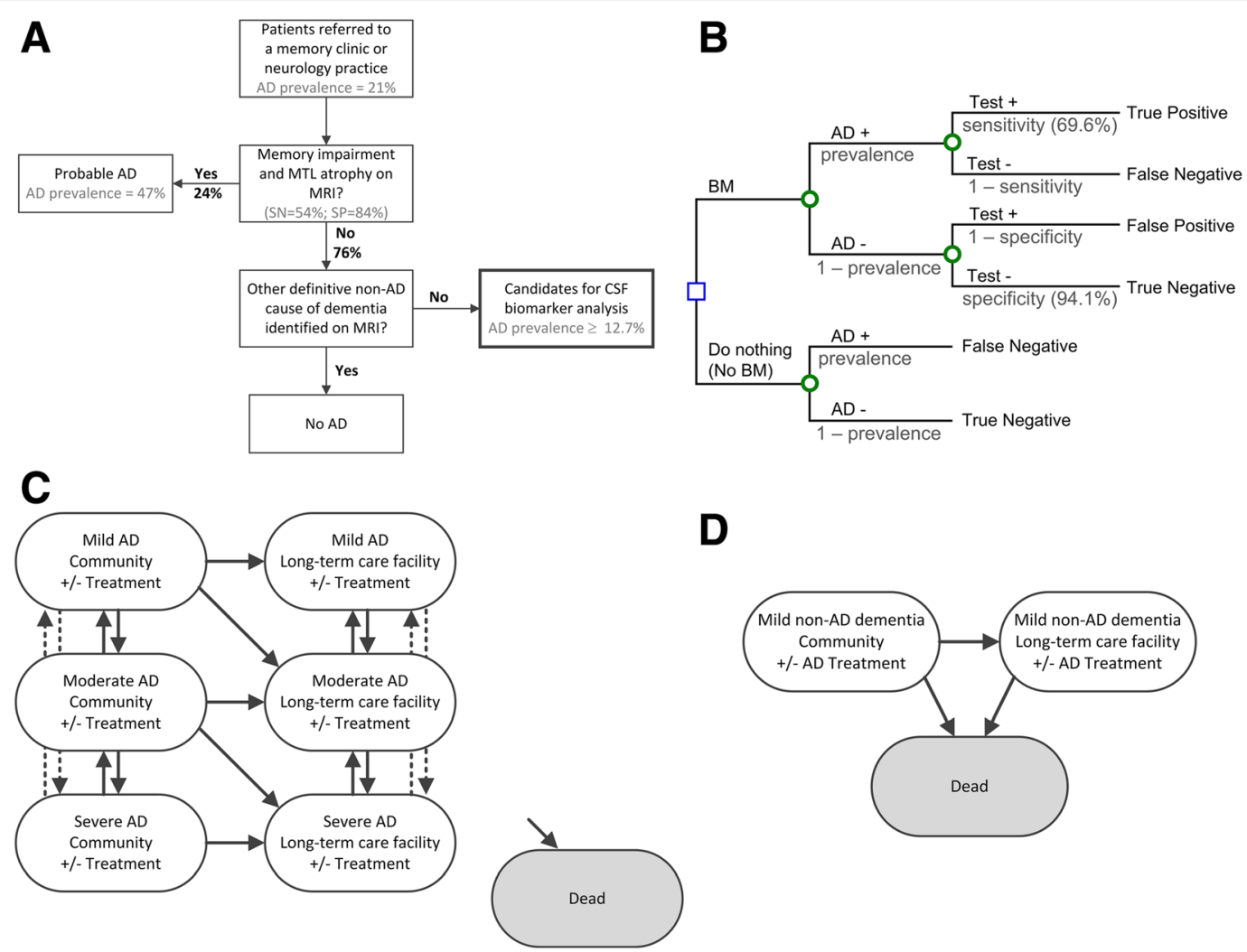

Fig. 1 Patient cohort and decision model. a Flowchart of patients referred to a memory clinic or neurology practice with suspected dementia, some of whom would be diagnosed with probable AD after clinical assessment and MRI. If MRI does not provide a definitive non-AD diagnosis for any patients, and if all patients remain candidates for biomarker analysis, the pretest prevalence of $A D$ is $12.7 \%$. If MRI provides a definitive non-AD diagnosis for some patients, the pretest prevalence of $A D$ in the cohort patients who continue to have suspected AD is greater than $12.7 \%$. b Schematic of a decision tree. The blue square represents the decision whether to use CSF biomarkers. Green circles represent chance events. The population is divided into four groups on the basis of whether the patients have Alzheimer's disease and the outcome of the diagnostic strategy: true-positive, false-negative, false-positive, and true-negative. The proportion of patients in each group is determined by the prevalence of AD in the evaluated cohort and the sensitivity and specificity of each diagnostic strategy. c Markov model of Alzheimer's disease. Patients begin in the health states for community-dwelling mild, moderate, and severe AD. Each month, patients may die, progress or regress in terms of disease severity, discontinue or reinitiate treatment, transition from living in the community to living in a long-term care facility, or stay in the same health state. In the model, patients living in a long-term care facility cannot return to living in the community. $\mathbf{d}$ Markov model for patients with non-AD dementia. Patients begin in the community-dwelling non-AD dementia state. Only those with a false-positive diagnosis of AD receive AD treatment. Each month, patients may die, discontinue or reinitiate treatment, transition from living in the community to living in a long-term care facility, or stay in the same health state. AD Alzheimer's disease, BM Biomarker, CSF Cerebrospinal fluid, MRI Magnetic resonance imaging, MTL Medial temporal lobe, SN Sensitivity, SP Specificity

the analysis of previously published model-based costeffectiveness studies of AD diagnosis [20, 25].

\section{Data and assumptions \\ Patient cohort}

The prevalence of $\mathrm{AD}$ in a cohort of patients with possible dementia varies across referral centers and increases with patient age and family history [1]. Of the 8495 patients referred to 30 U.S. Alzheimer's disease centers, $24 \%$ were diagnosed with mild AD [26]. We estimated the true prevalence to be $21 \%$, adjusting for the accuracy of diagnosis with clinical assessment and MRI (as the status quo), where proportion diagnosed = prevalence $\times$ sensitivity $+(1-$ prevalence $) \times(1-$ specificity $)$.
Clinical assessment and MTL atrophy seen on MRI would help identify approximately half of the patients with $\mathrm{AD}$ in the referral population (sensitivity of memory impairment plus MRI is 54\% [13]). Accounting for the diagnosis of $\mathrm{AD}$ after MRI, the approximate prevalence of $\mathrm{AD}$ in the remaining patients is $12.7 \%$ (Fig. 1a). In addition, MRI may provide another definitive diagnosis where the possibility of concomitant AD is highly unlikely and thus further consideration of $\mathrm{AD}$ using biomarkers is no longer clinically relevant. This patient selection will increase the pretest prevalence of $A D$ (by which we mean the probability of $\mathrm{AD}$ in the cohort of patients with memory impairment, no abnormal MTL atrophy, and no alternative diagnosis precluding $\mathrm{AD}$ ) in 
Table 1 Base case inputs, ranges for sensitivity analysis, and sources

\begin{tabular}{|c|c|c|c|c|}
\hline Parameter & Base case & Low value & High value & Source [reference] \\
\hline \multicolumn{5}{|l|}{ Patient population } \\
\hline Start age, years & 65 & 55 & 75 & [4] \\
\hline \multicolumn{5}{|c|}{ Initial AD severity distribution (\%) } \\
\hline Mild & 70 & 0.5400 & 0.783 & [67] \\
\hline Moderate & 28 & 0.1850 & 0.427 & [67] \\
\hline Severe & 2 & 0.0170 & 0.033 & [67] \\
\hline
\end{tabular}

Diagnosis

Diagnostic test accuracy

Status quo: clinical assessment plus MR neuroimaging (CA + MR)

$\begin{array}{lllll}\text { Sensitivity }\left(\mathrm{SN}_{\mathrm{MR}}\right) & 0.54 & 0.46 & 0.62 & \text { [13] } \\ \text { Specificity }\left(\mathrm{SP}_{\mathrm{MR}}\right) & 0.84 & 0.79 & 0.89 & \text { [13] }\end{array}$

Revised criteria: clinical assessment plus MR neuroimaging and/or biomarker analysis

$\begin{array}{lllll}\text { Sensitivity }\left(S N_{M R+B M}\right) & 0.86 & 0.80 & 0.92 & \text { [13] } \\ \left.\text { Specificity (SP } \mathrm{MR}_{\mathrm{MRM}}\right) & 0.79 & 0.74 & 0.84 & \text { [13] }\end{array}$

Diagnostic accuracy of CSF biomarkers in patients with no medial temporal lobe atrophy on MRI

$\begin{array}{lllll}\text { Sensitivity (SN } \mathrm{BM}_{\text {MR- }} \text { ) } & 0.698 & 0.54 & 0.86 & \text { Calculated }^{\text {a }} \\ \text { Specificity (SP } \text { BM|MR- } \text { ) } & 0.941 & 0.89 & 0.98 & \text { Calculated }^{\text {a }}\end{array}$

Biomarker analysis

$\begin{array}{lllll}\text { Cost } & 463 & 250 & 600 & {[50]} \\ \text { QALY toll } & -0.008 & 0 & -0.02 & {[19,57]}\end{array}$

AD natural history model

Mortality

Age-specific mortality due to causes other than AD

HRs for AD-specific mortality

$\begin{array}{lllll}\text { Mild } & 2.92 & 2.34 & 3.52 & \text { [29] } \\ \text { Moderate } & 3.85 & 2.94 & 5.05 & \text { [29] } \\ \text { Severe } & 9.52 & 6.60 & 13.4 & \text { [29] }\end{array}$

Disease progression without AD treatment (annual rate per 100,000)

From mild

$\begin{array}{lllll}\text { To moderate } & 27,710 & 24,939 & 30,481 & {[25,31]} \\ \text { To severe } & 1385 & 1247 & 1524 & {[25,31]}\end{array}$

From moderate

$\begin{array}{lllll}\text { To mild } & 4478 & 4030 & 4925 & {[25,31]} \\ \text { To severe } & 31,829 & 28,647 & 35,012 & {[25,31]}\end{array}$

From severe

To mild

$\begin{array}{llll}385 & 347 & 424 & {[25,31]}\end{array}$

To moderate

Transition to long-term care facility (annual rate per 100,000)

$\begin{array}{llll}2110 & 500 & 4000 & {[31,43,44]} \\ 6957 & 1500 & 8000 & {[31,43,44]} \\ 11,747 & 2500 & 15,000 & {[31,43,44]}\end{array}$

From moderate

$[25,31$

$[25,31]$

From severe 
Table 1 Base case inputs, ranges for sensitivity analysis, and sources (Continued)

$\mathrm{AD}$ treatment

Treatment uptake and adherence

Treatment initiation

Donepezil, at diagnosis

Memantine, at transition to severe $A D$

Treatment discontinuation (annual rate per 100,000)

Donepezil, community dwelling

Donepezil, long-term care facility dwelling

Memantine

0.45

0.36

0.27

0.22

28,768

10,536

51,083

12,783

30,111

Treatment reinitiation after quitting (annual rate per 100,000)

33,142

22,314

23,105

17,834

Memantine

Treatment effectiveness

Donepezil HRs

Transition from mild to moderate

Transition from moderate to mild

Transition from community to long-term care facility

Memantine

Incremental utility (annualized)

$H R$, transition from community to long-term care facility

Costs (US\$)

Age-specific baseline costs

$$
\begin{aligned}
& \text { 45-64 years } \\
& 65-84 \text { years } \\
& >84 \text { years }
\end{aligned}
$$

Annual incremental costs by disease severity (including costs of informal caregiving)

\section{Community dwelling}

Patients without $A D$

Mild AD

Moderate AD

Severe AD

Long-term care facility dwelling

Facility cost

Patients without AD

Mild AD

Moderate AD

Severe AD

Medication (annual)

Donepezil, 10 mg/day

Memantine, $10 \mathrm{mg} /$ day

Age-specific annual health care costs in the year of death

$<90$ years

$>90$ years

\section{4,128}

24,128

33,845

60,160

83,950

9872

9872

9872

9847

2473

3192

35,158

25,455
17,369

17,369

25,000

50,000

70,000

7000

7000

7000

7000

2000

2500

32,000

22,000
0.56

0.45

35,667

69,315

44,629

40,132

25,541

0.989

6.95

0.5

0.1

0.5

Estimated (see Methods)

[51]

[51]

[51]

$[37,39,41]$

[38]

[36]

[6]

[38]

[6]

[25]

[7]

8000

16,000

34,000
Assumed same as donepezil

30,369

Assumed the same as Mild AD

30,369

40,000

69,000

95,000

12,000

12,000

12,000

12,000

4288

5957

39,500

28,000 (see Additional file 1)

(see Additional file 1)

(see Additional file 1)

[52]

Assumed the same as Mild AD (see Additional file 1) (see Additional file 1) (see Additional file 1)

[69]

[69]

[70]

[70] 
Table 1 Base case inputs, ranges for sensitivity analysis, and sources (Continued)

\begin{tabular}{|c|c|c|c|c|}
\hline \multicolumn{5}{|l|}{ Utilities } \\
\hline \multicolumn{4}{|l|}{ Age-specific weights } & \multirow[t]{6}{*}[54,55]{} \\
\hline $60-64$ years & 0.83 & 0.822 & 0.835 & \\
\hline $65-69$ years & 0.82 & 0.820 & 0.826 & \\
\hline 70-74 years & 0.81 & 0.803 & 0.818 & \\
\hline $75-79$ years & 0.79 & 0.786 & 0.794 & \\
\hline$>79$ years & 0.74 & 0.730 & 0.742 & \\
\hline \multicolumn{5}{|l|}{ Health state-specific weights } \\
\hline \multicolumn{5}{|l|}{ Community dwelling } \\
\hline Patients without AD & 0.68 & 0.52 & 0.80 & Assumed same as mild AD \\
\hline Mild AD & 0.68 & 0.52 & 0.80 & {$[25]$} \\
\hline Moderate AD & 0.54 & 0.30 & 0.70 & {$[25]$} \\
\hline Severe AD & 0.37 & 0.25 & 0.50 & {$[25]$} \\
\hline \multicolumn{5}{|c|}{ Long-term care facility dwelling } \\
\hline Patients without AD & 0.71 & 0.55 & 0.80 & Assumed same as mild AD \\
\hline Mild AD & 0.71 & 0.55 & 0.80 & {$[25]$} \\
\hline Moderate AD & 0.48 & 0.30 & 0.60 & {$[25]$} \\
\hline Severe AD & 0.31 & 0.20 & 0.45 & {$[25]$} \\
\hline \multicolumn{5}{|c|}{ 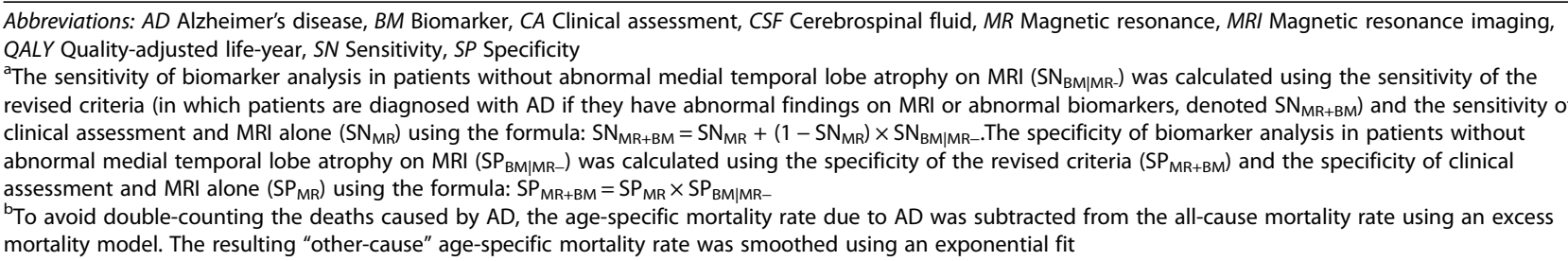 } \\
\hline
\end{tabular}

patients still considered candidates for biomarker analysis. Specifically, if $10 \%, 20 \%$, or $40 \%$ of non-AD patients are correctly identified as having an alternative diagnosis (and not having AD) after MRI, then the pretest prevalence of $\mathrm{AD}$ increases to $14 \%, 15 \%$, or $19 \%$, respectively. Furthermore, if patients without memory impairment are excluded, the prevalence of $\mathrm{AD}$ in the cohort of patients considered for biomarker analysis increases to $39 \%$ (the sensitivity and specificity of memory impairment alone are $93 \%$ and $68 \%$, respectively [13]). Variation in the case mix across referral centers, including the prevalence of $\mathrm{AD}$ and the distribution of causes for non-AD dementia, creates high uncertainty in the prevalence of $\mathrm{AD}$ in patients who remain without a definitive diagnosis after neuroimaging. Therefore, base case results are presented over the full range of possible AD prevalence.

\section{Diagnostic accuracy}

Bouwman et al. retrospectively evaluated the diagnostic accuracy of clinical assessment plus neuroimaging by $\mathrm{MR}$ and the revised AD diagnostic criteria [27] in 138 patients with $\mathrm{AD}$ and 223 memory clinic patients without $\mathrm{AD}$ [13]. Under the revised AD diagnostic criteria, patients were defined as having AD when clinical assessment indicated episodic memory impairment and either evidence of MTL atrophy and/or an abnormal biomarker profile $[13,27]$. MTL atrophy was scored visually on a scale of 0 (no atrophy) to 4 (severe atrophy) for both left and right hippocampi and then averaged to generate a single score. Positive $\mathrm{AD}$ findings were based on age-specific thresholds: $\geq 1$ was considered abnormal for patients aged $<65$ years; $\geq 1.5$ was considered abnormal for patients aged 65-75 years; and $>2$ was considered abnormal for patients $>75$ years of age. For CSF biomarker analysis, CSF was obtained using a standard lumbar puncture procedure and measured by commercially available sandwich enzyme-linked immunosorbent assays. Positive $\mathrm{AD}$ findings based on CSF biomarkers required at least two of the three biomarker criteria to be satisfied: low $\mathrm{A} \beta_{42}$ concentrations $(<495 \mathrm{ng} / \mathrm{L})$, increased total tau concentrations (>356 ng/L), or increased phospho-tau concentrations $(>54 \mathrm{ng} / \mathrm{L})$. (For further information, refer to Bouwman et al. [13].)

We calculated the sensitivity and specificity of biomarker analysis performed in patients without evidence of MTL atrophy on MRI by solving for the values that would achieve the overall sensitivity and specificity 
observed using the revised AD diagnostic criteria. In sensitivity analyses, we considered a wide range of values for biomarker sensitivity and specificity after a normal MRI, with sensitivity ranging from $54 \%$ to $86 \%$ (base case $69.6 \%$ ) and specificity ranging from $89 \%$ to $98 \%$ (base case $94.1 \%$ ).

\section{Mortality}

All-cause mortality was estimated using 2009 U.S. life tables [28]. To estimate the total mortality rate for a patient with $\mathrm{AD}$ at each stage of the disease, we multiplied the age-specific mortality rate for death due to other causes by AD severity-specific mortality HRs [29]. In our model, AD treatments did not influence mortality, because an analysis of the National Alzheimer's Coordinating Center (NACC) Uniform Data Set indicated that AD treatment did not influence the rate of death after adjusting for disease severity and other factors influencing treatment use [30].

\section{Natural history of $A D$}

Transition rates between $\mathrm{AD}$ severity health states and between living in the community to living in a long-term care facility (LTCF) were estimated using the NACC Uniform Data Set [31]. Despite the progressive nature of $\mathrm{AD}$, this analysis and a similar analysis of the Consortium to Establish a Registry for Alzheimer's Disease dataset estimated a positive probability of transitioning backward (e.g., from moderate to mild AD) [32]. Possible explanations for backward transition include variation in clinical presentation and assessment, concomitant disease, and treatment adjustments resulting in noisy observations over time or masking the true disease severity [32]. We used the severity-specific proportion of patients with $\mathrm{AD}$ on acetylcholinesterase inhibitor treatment and the HRs for progression on treatment to calculate treatment-stratified transition rates (details in Additional file 1: Section 1.1).

\section{Treatment regimens, adherence, and efficacy}

Treatment dosage and schedule were incorporated in accordance with various guidelines: donepezil $10 \mathrm{mg}$ per day in mild and moderate $\mathrm{AD}$ [33-35] and memantine $10 \mathrm{mg}$ per day in severe $\mathrm{AD}$ [33]. We represented all acetylcholinesterase inhibitors with donepezil because it is the most commonly prescribed of these drugs [36].

Acetylcholinesterase inhibitor uptake rates vary significantly across study cohorts, with initiation rates ranging from $27 \%$ [37] to $97 \%$ [38] in newly diagnosed patients with $\mathrm{AD}$ in the community. We estimated a moderate uptake rate of $45 \%$ on the basis of a study of communitydwelling patients who screened positive for dementia in a primary care setting [39]. Specialized or coordinated care increases treatment uptake rates [40]; therefore, we considered uptake rates from $27 \%$ to $56 \%$ in sensitivity analysis [37, 41]. Base case treatment discontinuation and reinitiation rates were informed by large observational cohorts such that $25 \%$ of community-dwelling patients and $46.4 \%$ of facility-dwelling patients discontinued AD treatment each year [36, 42], and $63 \%$ of communitydwelling patients and $36 \%$ of facility-dwelling patients who had discontinued $\mathrm{AD}$ treatments restarted treatment within 1 year $[6,38]$.

Consistent with previous model-based analyses of $\mathrm{AD}$, acetylcholinesterase inhibitor treatment reduced the transition rate from mild $\mathrm{AD}$ to moderate $\mathrm{AD}$ and increased the transition rate from moderate $\mathrm{AD}$ to mild $\mathrm{AD}$ [25]. The benefit of memantine treatment was incorporated into our model by an improved quality of life for patients with severe AD by 0.051 QALYs per year, which we estimated on the basis of average improvement in activities of daily living reported in a metaanalysis [7]. In the base case, consistent with previously published model-based analyses of AD treatment [25], we assumed that donepezil treatment does not reduce the rate of transition between moderate and severe disease, although we explored this possibility in sensitivity analysis. In the model, patients not on $\mathrm{AD}$ treatment are 2.7 times more likely to transition to an LTCF, as specifically reported by authors of a large U.S. medical claims database analysis including more than 5000 patients with AD [43] and consistent with other literature reports [31, 44-46].

In the base case analysis, patients without $\mathrm{AD}$ received no benefits from AD treatment, but we varied this assumption in sensitivity analysis. Occupational or psychosocial treatments were not included in the model, because they likely incur similar costs and provide benefits to patients with $\mathrm{AD}$ dementia and non-AD dementia [47-49].

\section{Costs}

We identified the clinical visit and laboratory testing codes with the Healthcare Common Procedure Coding System (HCPCS) and Current Procedural Terminology (CPT), then we estimated their cost using the 2013 Medicare reimbursement schedule [50]. We assumed biomarker analysis required a lumbar puncture procedure for the collection of CSF (CPT code 62270), an immunoassay analysis (HCPCS code 83520), and a follow-up visit with a neurologist in which the diagnosis is reported (CPT code 99213), resulting in a total cost of $\$ 463$.

In each month, individuals accrued age-specific health care costs unrelated to $\mathrm{AD}$, additional $\mathrm{AD}$ severityspecific health care costs, and location-specific (community or LTCF) supportive care costs (paid and unpaid). Agespecific health care costs unrelated to $\mathrm{AD}$, including out-of-pocket health care expenses, were based on the U.S. national average, which we smoothed using an exponential fit with a cap at the average annual cost of $\$ 33,870$ for patients aged 90 years and older [51]. AD 
severity-specific costs of inpatient care, outpatient care, emergency care, and unpaid caregiving are detailed in Additional file 1: Section 1.2. The annual cost of living in an LTCF was estimated to be $\$ 83,950$ (in 2013 U.S. dollars), based on the U.S. national average cost of a semiprivate room in a nursing home [52]. Costs were adjusted for inflation to constant 2013 U.S. dollars using the gross domestic product deflator [53].

\section{Quality of life}

We estimated baseline age-specific utilities from the Medical Expenditure Panel Survey data [54, 55]. Ageand $\mathrm{AD}$ severity-specific utilities were incorporated into the model by multiplying the age-specific utility by the AD severity-specific utility. Utility weights for each $A D$ disease state were estimated on the basis of a prior costeffectiveness analysis [25]. To our knowledge, no study to date has evaluated the one-time utility toll associated with embarrassment and discomfort before, during, and after a diagnostic test requiring lumbar puncture, including the risk and consequences of lumbar puncture-associated moderate to severe headache [56]. We assumed the onetime reduction in quality of life associated with lumbar puncture is approximately the same as the reduction in quality of life associated with breast biopsy, which has been measured to be equivalent to 2.92 quality-adjusted lifedays (annualized to a one-time toll of 0.008 QALY incurred at the time of the test) [57].

\section{Analysis}

We calculated the average lifetime discounted costs and QALYs for each diagnostic outcome and for each diagnostic strategy. If neither strategy cost less and provided more QALYs than the other, we calculated the incremental cost-effectiveness ratio (ICER). In a probabilistic analysis, we ran 10,000 independent simulations in which inputs were selected randomly from the probability distributions described in Additional file 1: Section 1.3 to determine $95 \%$ CIs for each outcome. We also performed deterministic sensitivity analyses to evaluate the robustness of our findings to uncertainty in model parameters and assumptions.

To provide general insight into the test characteristics that would make a new test or test combination both clinically and economically attractive after MRI, we identified the "challenge region" as described by Phelps and Mushlin at the willingness-to-pay (WTP) thresholds of $\$ 50,000$ and $\$ 100,000$ per QALY gained [58]. The boundary of the challenge region is identified as any set of new test characteristics, sensitivity $r_{1}$ and specificity $r_{2}$, for which the incremental net monetary benefit (INMB) compared with the current technology, with sensitivity $q_{1}$ and specificity $q_{2}$, at the WTP threshold (denoted $\lambda$ ) is greater than 0 . The INMB comparing the two tests is calculated as

$$
\begin{aligned}
\text { INMB } & =p\left(r_{1}-q_{1}\right)\left[\begin{array}{c}
\lambda\left(\mathrm{QALY}_{\text {TruePositive }}-\mathrm{QALY}_{\text {FalseNegative }}\right) \\
-\left(\text { Cost }_{\text {TruePositive }}-\text { Cost }_{\text {FalseNegative }}\right)
\end{array}\right] \\
& +(1-p)\left(r_{2}-q_{2}\right)\left[\begin{array}{c}
\lambda\left(\mathrm{QALY}_{\text {TrueNegative }}-\mathrm{QALY}_{\text {FalsePositive }}\right) \\
-\left(\text { Cost }_{\text {TrueNegative }}-\text { Cost }_{\text {FalsePositive }}\right)
\end{array}\right] \\
& -\Delta \text { TestCost }-\lambda \Delta \text { TestQALY }^{\text {Test }}
\end{aligned}
$$

where $p$ is the prevalence of the disease, $r_{1}-q_{1}$ is the improvement (or reduction) in sensitivity, $r_{2}-q_{2}$ is the improvement (or reduction) in specificity, $\left[\lambda\left(\right.\right.$ QALY $_{\text {TruePositive }}-$ QALY FalseNegative $\left._{\text {e }}\right)-\left(\right.$ Cost $_{\text {TruePositive }}-$ Cost $\left.\left._{\text {FalseNegative }}\right)\right]$ is the INMB of preventing a false-negative diagnosis, $\left[\lambda\left(\right.\right.$ QALY TrueNegative $\left.-\mathrm{QALY}_{\text {FalsePositive }}\right)-\left(\right.$ Cost $_{\text {TrueNegative }}-$ Cost $\left.\left._{\text {FalsePositive }}\right)\right]$ is the INMB of preventing a false-positive diagnosis, $\triangle$ TestCost is the difference in cost between the new and old diagnostic strategies, and $\triangle T$ TestQALY is the difference in the short-term quality-of-life effects associated with the test strategy.

\section{Results}

Lifetime costs and benefits of each diagnostic outcome

The lifetime discounted costs and QALYs associated with each possible diagnosis are shown in Table 2. Accurate diagnosis of $\mathrm{AD}$ decreased lifetime discounted costs by $\$ 9954$ and increased lifetime QALYs by 0.248 . In non-AD patients, a false diagnosis of $\mathrm{AD}$ increased lifetime costs by $\$ 11,345$ due to unnecessary treatment costs.

\section{Effectiveness and cost-effectiveness of diagnostic alternatives}

At a $12.7 \%$ pretest probability of $\mathrm{AD}$, biomarker analysis increased the average cost per patient by $\$ 165$ (95\% CI $-\$ 1865$ to $\$ 1625$ ) and increased the average QALYs per patient by 0.015 (95\% CI -0.011 to 0.051 ). The relatively small gain in QALYs was due primarily to the short-term discomfort associated with the lumbar puncture procedure (-0.008 QALY), which was experienced by all patients. At this pretest probability of $\mathrm{AD}$, the ICER of biomarker analysis was $\$ 11,032$ per QALY gained (Fig. 2a). Probabilistic analysis identified extremely high uncertainty: a $40 \%$ probability that biomarker analysis will decrease costs and increase QALYs, and a $7 \%$ probability that it will do the opposite (increase costs and decreased QALYs). Overall, at an expected pretest prevalence of $12.7 \%$, biomarkers were identified as cost-effective in $72 \%$ of simulations using a WTP threshold of $\$ 50,000$ per QALY gained and $82 \%$ of simulations using a WTP threshold of $\$ 100,000$ per QALY gained (Fig. 2b). 
Table 2 Average per-patient lifetime discounted costs and quality-adjusted life-years, by diagnostic outcome and strategy

\begin{tabular}{|c|c|c|c|c|}
\hline & Cost (U.S.\$) & LYS & QALYS & Probability of each outcome \\
\hline \multicolumn{5}{|c|}{ Lifetime discounted costs and benefits by diagnostic outcome } \\
\hline \multicolumn{5}{|l|}{$A D$} \\
\hline True-positive & $\$ 298,632$ & 6.781 & 2.916 & $8.9 \%$ \\
\hline False-negative & $\$ 308,586$ & 6.555 & 2.660 & $3.8 \%$ \\
\hline \multicolumn{5}{|l|}{ Not $A D$} \\
\hline False-positive & $\$ 294,732$ & 9.157 & 5.048 & $5.2 \%$ \\
\hline True-negative & $\$ 283,387$ & 9.157 & 5.048 & $82.1 \%$ \\
\hline \multicolumn{5}{|c|}{ Lifetime discounted costs and benefits by diagnostic strategy } \\
\hline Do nothing & $\$ 286,587(244,438$ to 337,270$)$ & & 4.745 (3.88 to 5.42$)$ & \\
\hline Biomarker analysis (BM) & $\$ 286,752(244,044$ to 337,163$)$ & & 4.760 (3.89 to 5.44$)$ & \\
\hline Incremental (BM vs. do nothing) & $\$ 165(-1865$ to 1625$)$ & & $0.015(-0.011$ to 0.051$)$ & \\
\hline \multicolumn{3}{|c|}{ Incremental cost-effectiveness ratio ( $\$$ per QALY gained) } & $\$ 11,032^{\mathrm{a}}$ & \\
\hline
\end{tabular}

Abbreviations: $A D$ Alzheimer's disease, $L Y$ Life-year, $Q A L Y$ Quality-adjusted life-year

${ }^{a}$ The empiric distribution of incremental cost-effectiveness ratios (ICERs) over the 10,000 simulations identified a $40 \%$ probability that biomarker analysis (BM) will decrease costs and increase QALYs and a 7\% probability that BM will increase costs and decrease QALYs, assuming an average AD prevalence of $12.7 \%$. Therefore, the $95 \% \mathrm{Cl}$ over the ICER ranges from BM is cost-saving to BM is dominated. Empiric $95 \%$ Cls were estimated from 10,000 simulations in which all input parameters were varied simultaneously

The results are highly influenced by the pretest prevalence of $\mathrm{AD}$ (Fig. 3a and b). The ICER rapidly increases as the pretest prevalence decreases (Fig. 3a); for pretest prevalence less than $9.1 \%$, biomarker analysis costs more than \$50,000 per QALY gained, and for pretest prevalence less than $7.5 \%$, biomarker analysis costs more than $\$ 100,000$ per QALY gained. For higher pretest prevalence, the ICER for biomarkers rapidly decreases, and for a pretest prevalence exceeding $15 \%$, the probability that biomarkers are cost-effective is $74 \%$, and deterministic analysis indicates biomarkers are cost-saving.

\section{Deterministic sensitivity analysis}

At a pretest prevalence greater than $9 \%$, deterministic sensitivity analysis indicated that biomarker analysis continued to be cost-effective within the ranges of uncertainty to disease progression rates, the rate of transition from living in the community to living in an LTCF, the cost of care in an LTCF, and the cost of biomarker testing. However, at a base case pretest prevalence of $12.7 \%$, our findings were sensitive to patient age, rate of transition into an LTCF, the costs of long-term care, test performance, and treatment adherence (Additional file 1: Table S3). High rates of AD treatment adherence decrease the cost-effectiveness of biomarker analysis because they increase the costs associated with false-positive diagnoses. However, in a sensitivity analysis in which we considered that $\mathrm{AD}$ treatment may provide $50 \%$ of the benefit to patients with a false-positive diagnosis $[59,60]$, biomarker analysis costs more than $\$ 50,000$ per QALY gained.
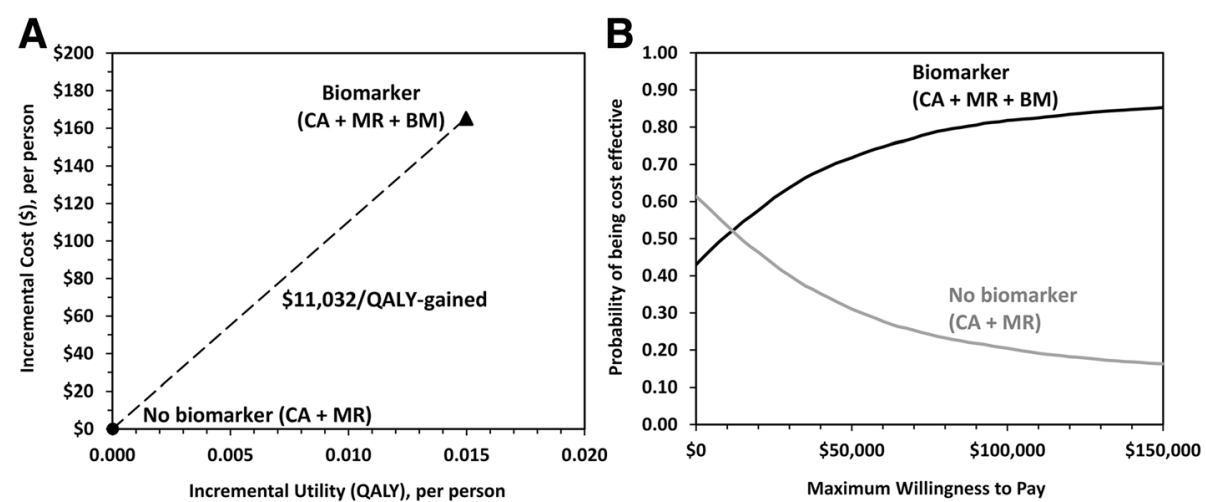

Fig. 2 a Cost-effectiveness plane: incremental costs and incremental QALYS of CSF biomarker analysis. b Probability of each diagnostic strategy being the cost-effective alternative at various willingness-to-pay thresholds when all model input parameters are varied simultaneously. AD Alzheimer's disease, BM Biomarker, CA Clinical assessment, MR Magnetic resonance, QALY Quality-adjusted life-year 
We relied heavily on the study of Bouwman et al. to estimate the sensitivity and specificity of biomarker analysis [13]. However, this study had relatively small sample size and used a gold standard of multidisciplinary team consensus rather than autopsy, the only true gold standard in AD diagnosis [61]. As such, we considered a wide range of sensitivities and specificities lower than in our base case (Additional file 1: Table S3). At moderately lower diagnostic accuracy (sensitivity 62\%, specificity $92 \%$ ), biomarker analysis remains the preferred alternative. At a low diagnostic accuracy (sensitivity 54\%, specificity $89 \%$ ), the ICER of biomarker analysis increases to $\$ 87,000$ per QALY gained. Lowering the specificity further (sensitivity $54 \%$, specificity $84 \%$ ), the ICER of biomarker analysis exceeds $\$ 100,000$ per QALY gained. Additionally, there is uncertainty about the proportion of patients who would receive a definitive non-AD diagnosis prior to biomarker analysis, which would increase the pretest prevalence of $\mathrm{AD}$ in the tested cohort. In this case of very low test accuracy, if $\mathrm{AD}$ prevalence in the tested cohort is $15 \%$, the ICER is $\$ 87,600$ per QALY gained.

Two-way sensitivity analysis of prevalence and age revealed that, for younger patients, biomarker analysis is cost-effective at pretest probabilities of AD less than $8 \%$ at WTP of $\$ 50,000$ per QALY gained (Fig. 4a). For older

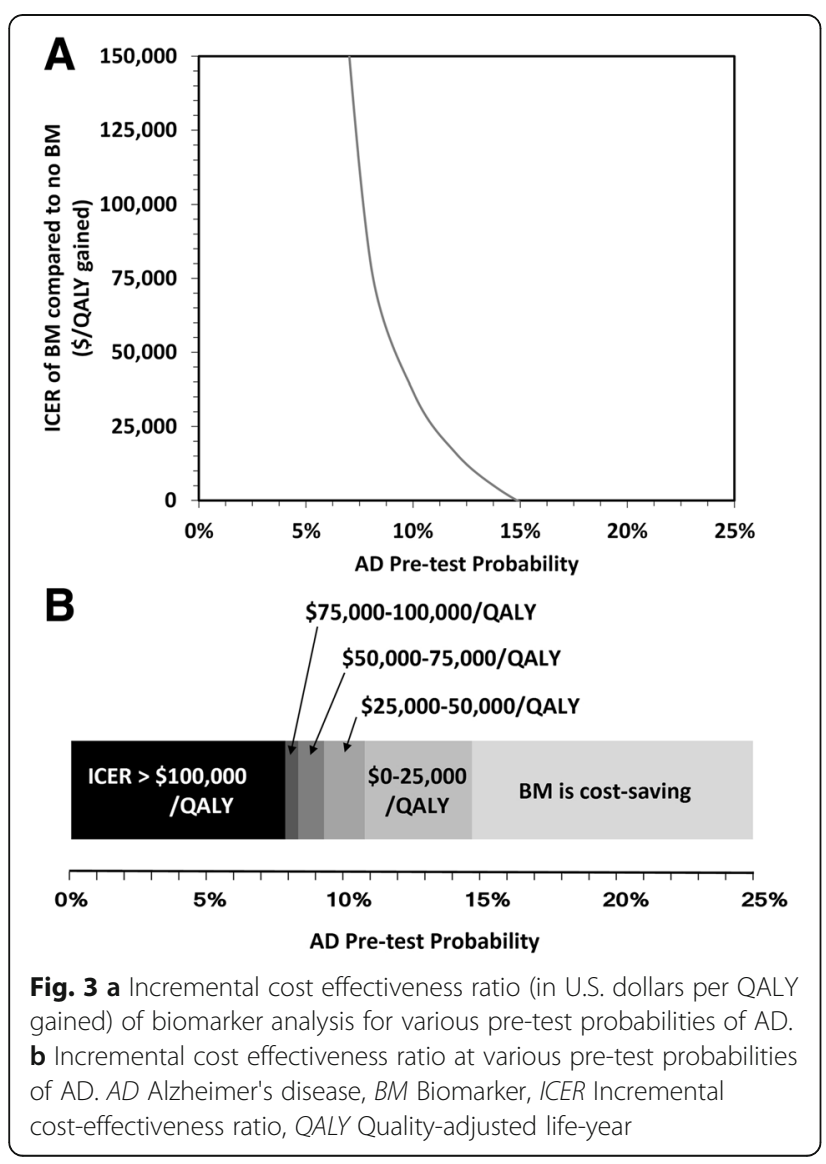

patients, such as those over the age of 75 years, biomarker analysis is cost-effective only in highly selected patient cohorts with pretest prevalence $>27 \%$ and $>20 \%$ at WTP of $\$ 50,000$ or $\$ 100,000$ per QALY gained, respectively (i.e., those with memory impairment). Two-way sensitivity analysis also identified that either increasing the cost of biomarker analysis by $\$ 1400$ or increasing the utility decrement by 0.020 QALYs was sufficient for biomarker analysis to no longer be cost-effective (Fig. 4b).

Structural sensitivity analysis on the natural history of non-AD patients indicated that biomarker analysis is slightly more cost-effective if the conditions affecting patients without $\mathrm{AD}$ are more severe than we assumed in our base case. Biomarker analysis is less cost-effective if patients without AD but who are falsely diagnosed with $\mathrm{AD}$ receive a small benefit from acetylcholinesterase inhibitor treatment (Additional file 1: Table S3). Biomarker analysis is also less cost-effective if correction is made when disease progresses for patients with initially falsenegative results (Additional file 1: Table S3).

\section{Challenge region}

When developing a diagnostic test, a trade-off exists between test sensitivity and specificity. In the case of $\mathrm{AD}$, improved test sensitivity prevents delay in access to quality-of-life treatments caused by false-negative diagnoses (valued at \$9954 per false-negative avoided), and improved test specificity prevents unnecessary treatment resulting from false-positive diagnoses (valued at $\$ 11,345$ per false-positive avoided). The challenge region presented in Fig. 5 identifies the collection of all sensitivity and specificity pairs where a hypothetical test, with a cost and short-term disutility similar to those of CSF biomarkers, would be cost-effective compared with no test at four levels of pretest prevalence: $7.5 \%, 12.7 \%, 15 \%$, and $30 \%$.

\section{Discussion}

For biomarker analysis to be cost-effective at a WTP of $\$ 50,000$ per QALY gained, the pretest prevalence of AD in the tested cohort must be more than 1 in 11 patients. Overall prevalence of $\mathrm{AD}$ in the referral population varies substantially across referral centers, with specialized centers diagnosing $\mathrm{AD}$ in approximately one-fourth of referred patients [26, 61]. Evaluation of MTL atrophy by MRI will diagnose at least half of patients with AD. MRI may also identify a definitive diagnosis other than $A D$, which may preclude the need for continued evaluation in some patients. The optimal policy may therefore vary across clinics and may further depend on specific patient risk factors. In patients presenting to memory clinics with memory impairment without MTL atrophy, AD pretest prevalence may be greater than 14.5\%; in these patients, biomarker analysis has the potential to be cost-saving. In addition to the benefits measured in the present study, 


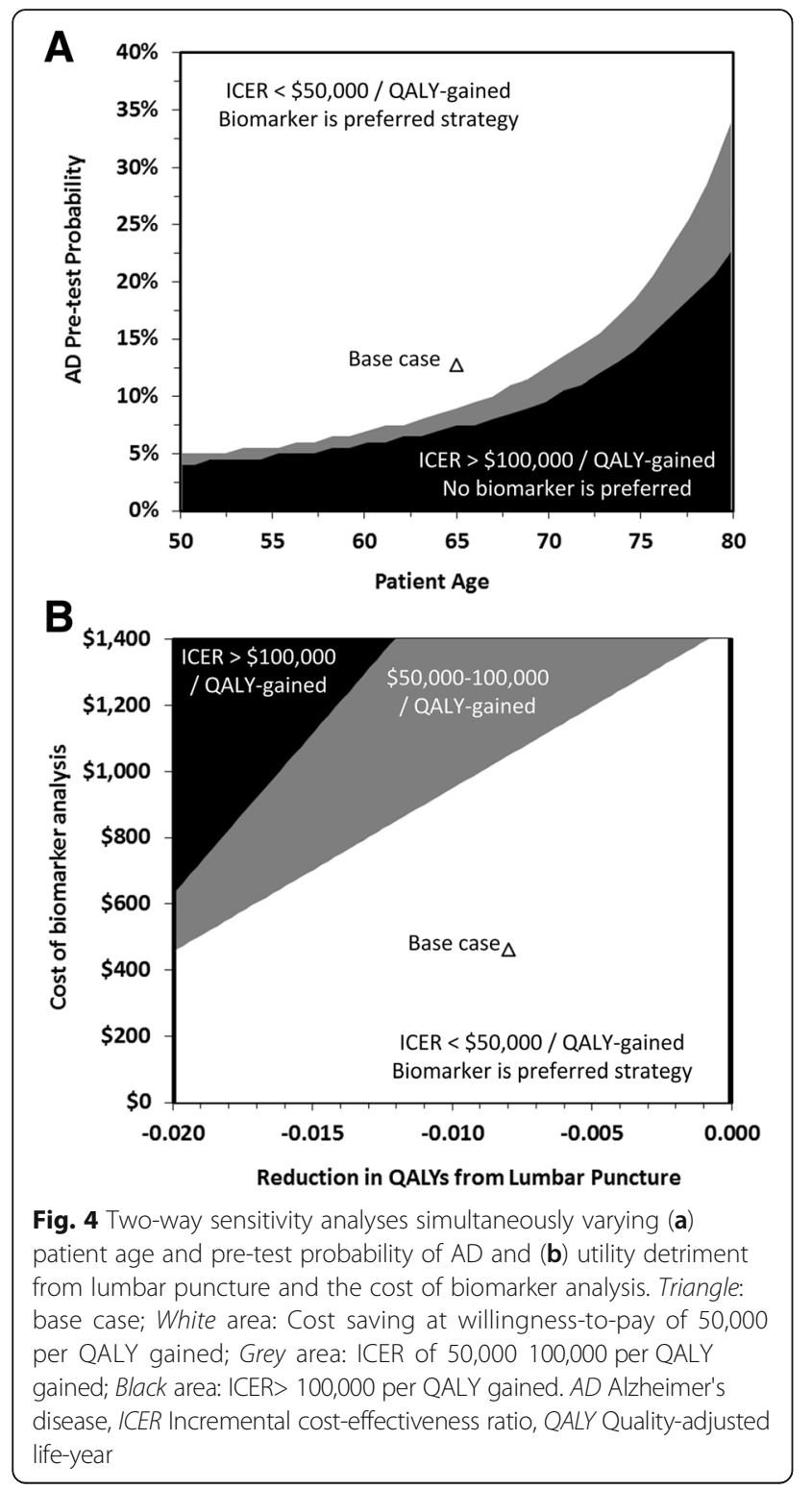

timely diagnosis would also enable patients and their families to make informed decisions in planning future caregiving at a time when all parties achieve the greatest benefit and enable patients to have a greater role in making their own health care decisions before cognitive impairment interferes [62].

In practice, treatment uptake and adherence are low [63]. However, even with very low rates of treatment uptake and high rates of treatment discontinuation, biomarker analysis remains the preferred alternative (Additional file 1: Table S3). However, if patients without $\mathrm{AD}$ who receive a false-positive diagnosis of $\mathrm{AD}$ (and therefore initiate treatment at the same rates as patients with a true-positive diagnosis of $\mathrm{AD}$ ) receive moderate benefits from donepezil and memantine for a disease with a similar progression to $\mathrm{AD}$,

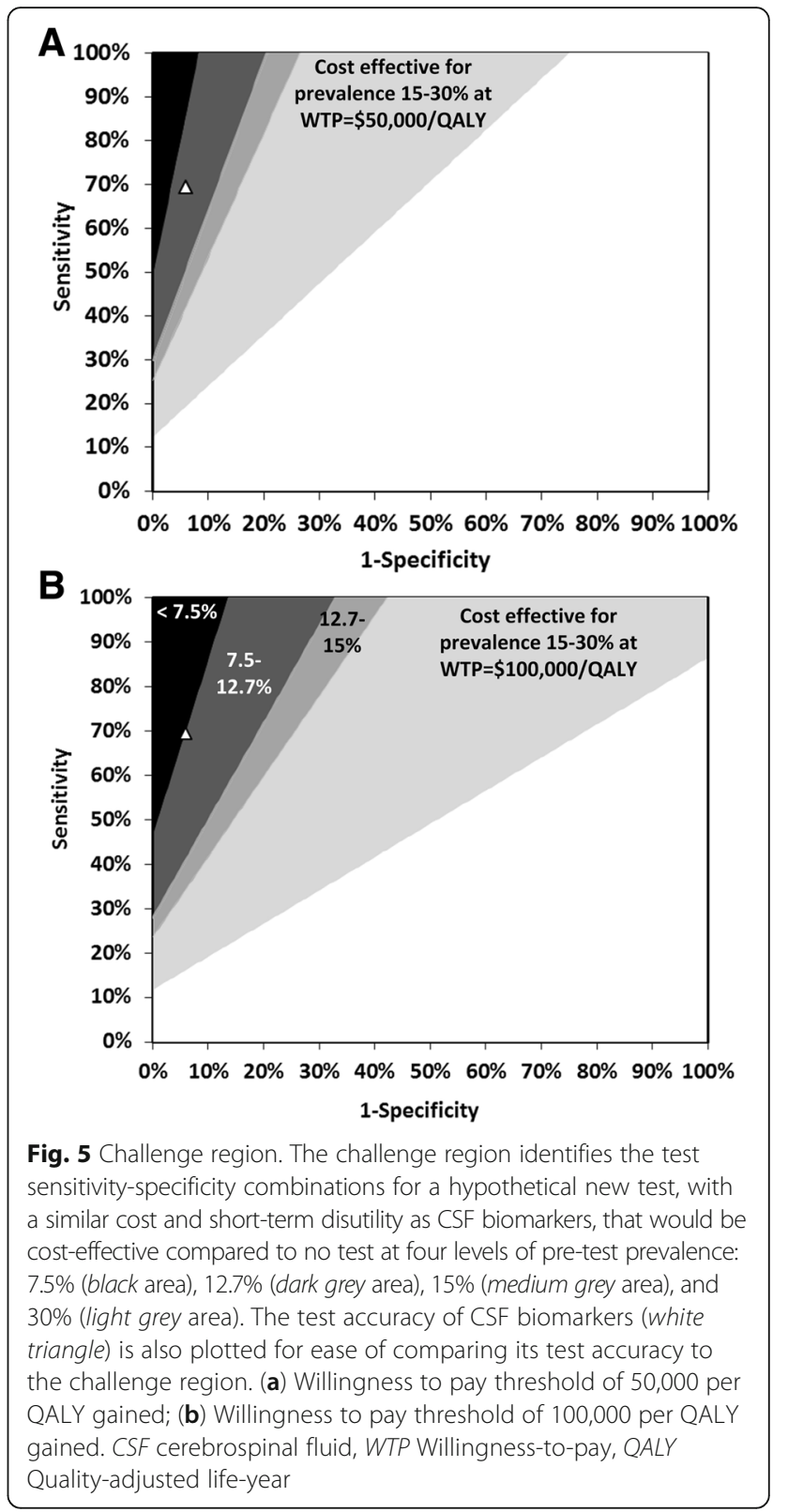

biomarker analysis is no longer the cost-effective option at a WTP of $\$ 50,000$ per QALY gained (Additional file 1: Table S3). This finding indicates that if patients with a false-positive diagnosis, for whom the cost of treatment will be incurred, receive a benefit from that treatment, the economic benefit derived from reducing the number of falsepositives decreases. This finding does not indicate that donepezil or memantine treatment for patients without $\mathrm{AD}$ is necessarily cost-effective. The cost-effectiveness of cholinesterase inhibitor treatment in patients with non-AD disease has been demonstrated for Lewy body dementia [64], but acetylcholinesterase inhibitors have not shown clinical benefit for patients with MCI [65]. 
In general, the cost-effectiveness of a treatment depends on the natural history of the disease as well as the cost and efficacy of all treatment alternatives available to patients with that condition.

Studies in which researchers have estimated the diagnostic accuracy of clinical assessment, neuroimaging, and CSF biomarkers vary widely in their findings [66]. We used the study by Bouwman et al., who retrospectively applied each potential diagnostic strategy to 138 patients with $\mathrm{AD}$ and 223 memory clinic patients without $\mathrm{AD}$ [13]. Relying on a single study provided internally consistent estimates for the sensitivity and specificity of each test and the tests compared with each other, which may not have occurred had we collected test accuracy information from independent studies performed with different patient populations. At low pretest probabilities $(<9 \%)$, the incremental cost of biomarker analysis was not robust to the uncertainty in test accuracy or many other input parameters. However, at higher pretest probabilities, the finding that biomarkers are cost-effective is robust to uncertainty in biomarker test accuracy (Fig. 5). This is relevant because specificity in particular may vary across referral centers, depending on the mix of patients composing the non-AD cohort. Greater confidence in the accuracy of diagnostic strategies can be established with larger sample size studies similar in design to that of Bouwman et al., in which multiple diagnostic criteria were applied to the same patients [13].

Our analysis has limitations, including a limited number of health states that do not fully represent the complex and multifaceted nature of $\mathrm{AD}$ and other neurological or psychiatric diseases represented in the non-AD population [18]. However, in addition to modeling cognitive functional decline, we included whether the patient was dwelling in the community or in an LTCF to incorporate elements of functional dependence, and we included disease severity-specific unpaid caregiving. Our inputs were derived from the medical literature. Specifically, transition rates for $\mathrm{AD}$ progression were based on an observational cohort not stratified by treatment status. In addition, several model parameters, including the accuracy of both diagnostic strategies, relied on studies with relatively small sample sizes and AD diagnosis based on clinical assessment, not on autopsy.

\section{Conclusions}

Biomarker testing reduces the number of false-negative diagnoses and therefore connects patients to treatment earlier, improving their quality of life. Although the costeffectiveness of biomarker analysis depends critically on the prevalence of $\mathrm{AD}$ in the tested population, it is costeffective at a WTP of $\$ 50,000$ per QALY gained in patient cohorts in which at least $1(9 \%)$ in 11 patients has AD. In patients presenting to memory clinics with memory impairment without neuroimaging evidence of MTL atrophy, AD prevalence likely exceeds $15 \%$. Biomarker analysis is potentially cost-saving and should be considered for adoption in high-prevalence centers.

\section{Additional file}

Additional file 1: Cost-effectiveness of cerebrospinal biomarkers for Alzheimer's diagnosis: supplemental Methods, Results, figures and tables as referenced in the text. (DOCX $91 \mathrm{~kb})$

\section{Abbreviations}

AD: Alzheimer's disease; A $\beta$ : Amyloid- $\beta$; BM: Biomarker; $C A$ : Clinical assessment; CPT: Current Procedural Terminology; CSF: Cerebrospinal fluid; CT: Computed tomography; HCPCS: Healthcare Common Procedure Coding System; ICER: Incremental cost-effectiveness ratio; INMB: Incremental net monetary benefit; LTCF: Long-term care facility; LY: Life-year; MCI: Mild cognitive impairment; MR: Magnetic resonance; MRI: Magnetic resonance imaging; MTL: Medial temporal lobe; NACC: National Alzheimer's Coordinating Center; PET: Positron emission tomography; QALY: Quality-adjusted life-year; SN: Sensitivity; SP: Specificity; SPECT: Single-photon emission computed tomography; WTP: Willingness to pay

\section{Acknowledgements}

Not applicable.

Funding

Funding was provided by the Natural Sciences and Engineering Research Council of Canada (NSERC).

\section{Availability of data and materials} Not applicable.

\section{Authors' contributions}

SAWL conceived of and designed the study, acquired data, analyzed and interpreted the results, drafted the manuscript, and gave final approval of the submitted manuscript. LAS conceived of and designed the study, interpreted the results, critically revised the manuscript for intellectual content, and gave final approval of the submitted manuscript. $\mathrm{VH}$ conceived of and designed the study, interpreted the results, critically revised the manuscript for intellectual content, and gave final approval of the submitted manuscript. LEC conceived of and designed the study, acquired data, analyzed and interpreted the results, critically revised the manuscript for intellectual content, and gave final approval of the submitted version.

\section{Competing interests}

The authors declare that they have no competing interests.

Consent for publication

All authors have approved the manuscript for submission.

Ethics approval and consent to participate

Not applicable.

\section{Author details}

${ }^{1}$ Ivey Business School, Western University, 1255 Western Road, London, ON N6G 0N1, Canada. ${ }^{2}$ School of Medicine, University College Cork, College Road, Cork T12 YN60, Ireland. 'epartment of Clinical Neurological Sciences, London Health Sciences Centre, Western University, London, ON N6A 5A5, Canada. ${ }^{4}$ Stroke, Dementia \& Heart Disease Laboratory, Western University, London, ON N6A 5A5, Canada. ${ }^{5}$ Department of Anatomy \& Cell Biology, Western University, London, ON N6A 5A5, Canada. ${ }^{6}$ Department of Biostatistics and Epidemiology, Schulich School of Medicine and Dentistry, Western University, London, ON N6A 5C1, Canada. 


\section{Received: 23 September 2016 Accepted: 8 February 2017} Published online: 16 March 2017

\section{References}

1. Alzheimer's Association. Alzheimer's disease facts and figures. Alzheimers Dement. 2014;10:e47-92.

2. Prince $M$, Bryce R, Albanese E, Wimo A, Ribeiro W, Ferri CP. The global prevalence of dementia: a systematic review and metaanalysis. Alzheimers Dement. 2013;9:63-75.e2

3. Trojanowski JQ, Hampel H. Neurodegenerative disease biomarkers: guideposts for disease prevention through early diagnosis and intervention. Prog Neurobiol. 2011;95:491-5.

4. Hebert LE, Weuve J, Scherr PA, Evans DA. Alzheimer disease in the United States (2010-2050) estimated using the 2010 census. Neurology. 2013;80: 1778-83.

5. Hunter CA, Kirson NY, Desai U, Cummings AKG, Faries DE, Birnbaum HG. Medical costs of Alzheimer's disease misdiagnosis among US Medicare beneficiaries. Alzheimers Dement. 2015;11:887-95.

6. Vidal JS, Lacombe JM, Dartigues JF, Pasquier F, Robert P, Tzourio C, et al. Memantine therapy for Alzheimer disease in real-world practice: an observational study in a large representative sample of French patients. Alzheimer Dis Assoc Disord. 2008;22:125-30.

7. McShane R, Areosa Sastre A, Minakaran N. Memantine for dementia. Cochrane Database Syst Rev. 2006;2:CD003154

8. Geldmacher DS, Kirson NY, Birnbaum HG, Eapen S, Kantor E, Cummings AK, et al. Implications of early treatment among Medicaid patients with Alzheimer's disease. Alzheimers Dement. 2014;10:214-24.

9. López-Bastida J, Hart W, García-Pérez L, Linertová R. Cost-effectiveness of donepezil in the treatment of mild or moderate Alzheimer's disease. $J$ Alzheimers Dis. 2009;16:399-407.

10. Feldman H, Gauthier S, Hecker J, Vellas B, Hux M, Xu Y, et al. Economic evaluation of donepezil in moderate to severe Alzheimer disease. Neurology. 2004:63:644-50.

11. Weycker D, Taneja C, Edelsberg J, Erder MH, Schmitt FA, Setyawan J, et al. Cost-effectiveness of memantine in moderate-to-severe Alzheimer's disease patients receiving donepezil. Curr Med Res Opin. 2007;23:1187-97.

12. Beach TG, Monsell SE, Phillips LE, Kukull W. Accuracy of the clinical diagnosis of Alzheimer disease at National Institute on Aging Alzheimer disease centers, 2005-2010. J Neuropathol Exp Neurol. 2012:71:266-73.

13. Bouwman FH, Verwey NA, Klein M, Kok A, Blankenstein MA, Sluimer JD, et al. New research criteria for the diagnosis of Alzheimer's disease applied in a memory clinic population. Dement Geriatr Cogn Disord. 2010;30:1-7.

14. Scheltens P, Blennow K, Breteler MMB, de Strooper B, Frisoni GB, Salloway S, et al. Alzheimer's disease. Lancet. 2016;388:505-17.

15. Bloudek LM, Spackman DE, Blankenburg M, Sullivan SD. Review and metaanalysis of biomarkers and diagnostic imaging in Alzheimer's disease. J Alzheimer's Dis. 2011;26:627-45.

16. Morris E, Chalkidou A, Hammers A, Peacock J, Summers J, Keevil S. Diagnostic accuracy of ${ }^{18} \mathrm{~F}$ amyloid PET tracers for the diagnosis of Alzheimer's disease: a systematic review and meta-analysis. Eur J Nucl Med Mol Imaging. 2016:43:374-85

17. Handels RL, Wolfs CA, Aalten P, Joore MA, Verhey FR, Severens JL. Diagnosing Alzheimer's disease: a systematic review of economic evaluations. Alzheimers Dement. 2014:10:225-37.

18. Green C, Shearer J, Ritchie CW, Zajicek JP. Model-based economic evaluation in Alzheimer's disease: a review of the methods available to model Alzheimer's disease progression. Value Health. 2011;14:621-30.

19. McMahon PM, Araki SS, Neumann PJ, Harris GJ, Gazelle GS. Costeffectiveness of functional imaging tests in the diagnosis of Alzheimer disease. Radiology. 2000;217:58-68.

20. McMahon PM, Araki SS, Sandberg EA, Neumann PJ, Gazelle GS. Cost-effectiveness of PET in the diagnosis of Alzheimer disease. Radiology. 2003;228:515-22.

21. Hornberger J, Michalopoulos S, Dai M, Andrade P, Dilla T, Happich M. Costeffectiveness of florbetapir-PET in Alzheimer's disease: a Spanish societal perspective. J Ment Health Policy Econ. 2015;18:63-73.

22. Valcárcel-Nazco C, Perestelo-Pérez L, Molinuevo JL, Mar J, Castilla I, SerranoAguilar P. Cost-effectiveness of the use of biomarkers in cerebrospinal fluid for Alzheimer's disease. J Alzheimers Dis. 2014;42:777-88.

23. Gold MR, Siegel JE, Russell LB, Weinstein MC, editors. Cost-effectiveness in health and medicine. 1st ed. London: Oxford University Press; 1996.
24. Weinstein MC, Skinner JA. Comparative effectiveness and health care spending —implications for reform. N Engl J Med. 2010;362:460-5.

25. Neumann PJ, Hermann RC, Kuntz KM, Araki SS, Duff SB, Leon J, et al. Costeffectiveness of donepezil in the treatment of mild or moderate Alzheimer's disease. Neurology. 1999;52:1138-45.

26. Steenland K, MacNeil J, Bartell S, Lah J. Analyses of diagnostic patterns at 30 Alzheimer's disease centers in the US. Neuroepidemiology. 2010;35:19-27.

27. Dubois B, Feldman HH, Jacova C, DeKosky ST, Barberger-Gateau P, Cummings J, et al. Research criteria for the diagnosis of Alzheimer's disease: revising the NINCDS-ADRDA criteria. Lancet Neurol. 2007;6:734-46.

28. Arias E. United States life tables, 2009. Natl Vital Stat Rep. 2014;62(7)

29. Andersen K, Lolk A, Martinussen T, Kragh-Sørensen P. Very mild to severe dementia and mortality: a 14-year follow-up - the Odense study. Dement Geriatr Cogn Disord. 2010;29:61-7.

30. Bloudek LM, Spackman DE, Veenstra DL, Sullivan SD. CDR state transition probabilities in Alzheimer's disease with and without cholinesterase inhibitor intervention in an observational cohort. J Alzheimer's Dis. 2011:24:599-607.

31. Spackman D, Kadiyala S, Neumann PJ, Veenstra D, Sullivan S. Measuring Alzheimer disease progression with transition probabilities: estimates from NACC-UDS. Curr Alzheimer Res. 2012;9:1050-8.

32. Neumann PJ, Araki SS, Arcelus A, Longo A, Papadopoulos G, Kosik KS, et al. Measuring Alzheimer's disease progression with transition probabilities. Neurology. 2001:57:957-64.

33. APA Work Group on Alzheimer's Disease and other Dementias. American Psychiatric Association practice guideline for the treatment of patients with Alzheimer's disease and other dementias, second edition. Am J Psychiatry. 2007;164(12 Suppl):1-56

34. Winslow BT, Onysko MK, Stob CM, Hazlewood KA. Treatment of Alzheimer disease. Am Fam Physician. 2011:83:1403-12.

35. Massoud F, Leger GC. Pharmacological treatment of Alzheimer disease. Can J Psychiatry. 2011;56:579-88.

36. Mucha L, Wang SS, Cuffel B, McRae T, Mark TL, del Valle M. Comparison of cholinesterase inhibitor utilization patterns and associated health care costs in Alzheimer's disease. J Manag Care Pharm. 2008;14:451-61.

37. van den Bussche $\mathrm{HH}$. Antidementia drug prescription sources and patterns after the diagnosis of dementia in Germany: results of a claims data-based 1-year follow-up. Int Clin Psychopharmacol. 2011;26:225-31.

38. Taipale H, Tanskanen A, Koponen M, Tolppanen AM, Tiihonen J, Hartikainen S. Antidementia drug use among community-dwelling individuals with Alzheimer's disease in Finland: a nationwide register-based study. Int Clin Psychopharmacol. 2014;29:216-23.

39. Michalowsky B, Eichler T, Thyrian JR, Hertel J, Wucherer D, Hoffmann W, et al. Healthcare resource utilization and cost in dementia: are there differences between patients screened positive for dementia with and those without a formal diagnosis of dementia in primary care in Germany? Int Psychogeriatr. 2016;28:359-69.

40. Wübbeler M, Wucherer D, Hertel J, Michalowsky B, Heinrich S, Meyer S, et al Antidementia drug treatment in dementia networks in Germany: use rates and factors associated with treatment use. BMC Health Serv Res. 2015;15:205.

41. Matthews FE, Muniz-Terrera G, McKeith I, Brayne C. Who will be eligible? An investigation of the dementia population eligible for cholinesterase treatment following the change in NICE guidance. Int J Geriatr Psychiatry. 2010;25:719-24.

42. Dybicz SB, Keohane DJ, Erwin WG, McRae T, Shah SN. Patterns of cholinesterase-inhibitor use in the nursing home setting: a retrospective analysis. Am J Geriatr Pharmacother. 2006:4:154-60.

43. Beusterien KM, Thomas SK, Gause D, Kimel M, Arcona S, Mirski D. Impact of rivastigmine use on the risk of nursing home placement in a US sample. CNS Drugs. 2004;18:1143-8.

44. Feldman HH, Pirttila T, Dartigues JF, Everitt B, Van Baelen B, Schwalen S, et al. Treatment with galantamine and time to nursing home placement in Alzheimer's disease patients with and without cerebrovascular disease. Int J Geriatr Psychiatry. 2009:24:479-88.

45. Geldmacher DS, Provenzano G, McRae T, Mastey V, leni JR. Donepezil is associated with delayed nursing home placement in patients with Alzheimer's disease. J Am Geriatr Soc. 2003;51:937-44.

46. Wattmo C, Wallin AK, Londos E, Minthon L. Risk factors for nursing home placement in Alzheimer's disease: a longitudinal study of cognition, ADL, service utilization, and cholinesterase inhibitor treatment. Gerontologist. 2011;51:17-27.

47. Søgaard R, Sørensen J, Waldorff FB, Eckermann A, Buss DV, Phung KTT, et al. Early psychosocial intervention in Alzheimer's disease: cost utility evaluation 
alongside the Danish Alzheimer's Intervention Study (DAISY). BMJ Open. 2014:4:e004105.

48. Oyebode JR, Parveen S. Psychosocial interventions for people with dementia: an overview and commentary on recent developments. Dementia (London). doi:10.1177/1471301216656096.

49. Olazarán J, Reisberg B, Clare L, Cruz I, Peña-Casanova J, Del Ser T, et al. Nonpharmacological therapies in Alzheimer's disease: a systematic review of efficacy. Dement Geriatr Cogn Disord. 2010;30:161-78.

50. Centers for Medicare \& Medicaid Services. Medicare fee-for-service payment schedule. 2009. https://www.cms.gov/Medicare/Medicare-Fee-for-ServicePayment/FeeScheduleGenInfo/index.html?redirect=/feeschedulegeninfo/. Accessed 16 July 2014

51. Lassman D, Hartman M, Washington B, Andrews K, Catlin A. US health spending trends by age and gender: selected years 2002-10. Health Aff (Millwood). 2014;33:815-22.

52. MetLife. 2012 MetLife market survey of long-term care costs. New York: Author; 2012. https://www.metlife.com/mmi/research/2012-market-surveylong-term-care-costs.html\#keyfindings. Accessed 16 July 2014.

53. Centers for Disease Control and Prevention (CDC). Health Data Interactive (HDI): mortality and life expectancy: mortality by underlying and multiple cause, ages 18+: US, 1981-2010. Atlanta: CDC; 2014. http://www.healthdata. gov/dataset/health-data-interactive-hdi. Accessed 19 Aug 2014.

54. Nyman JA, Barleen NA, Dowd BE, Russell DW, Coons SJ, Sullivan PW. Quality-oflife weights for the US population: self-reported health status and priority health conditions, by demographic characteristics. Med Care. 2007;45:618-28.

55. Sullivan PW, Ghushchyan V. Preference-based EQ-5D index scores for chronic conditions in the United States. Med Decis Making. 2006;26:410-20.

56. Thomas SR, Jamieson DR, Muir KW. Randomised controlled trial of atraumatic versus standard needles for diagnostic lumbar puncture. BMJ. 2000;321:986-90

57. Swan JS, Kong CY, Lee JM, Akinyemi O, Halpern EF, Lee PA, et al. Patient and societal value functions for the Testing Morbidities Index. Med Decis Making. 2013;33:819-38.

58. Phelps CE, Mushlin Al. Focusing technology assessment using medical decision theory. Med Decis Making. 1988:8:279-89.

59. Román GC, Salloway S, Black SE, Royall DR, DeCarli C, Weiner MW, et al. Randomized, placebo-controlled, clinical trial of donepezil in vascular dementia: differential effects by hippocampal size. Stroke. 2010;41:1213-21.

60. Malouf R, Birks J. Donepezil for vascular cognitive impairment. Cochrane Database Syst Rev. 2004;1:CD004395.

61. de Jager CA, Honey TE, Birks J, Wilcock GK. Retrospective evaluation of revised criteria for the diagnosis of Alzheimer's disease using a cohort with post-mortem diagnosis. Int J Geriatr Psychiatry. 2010;25:988-97.

62. Brooker D, La Fontaine J, Evans S, Bray J, Saad K. Public health guidance to facilitate timely diagnosis of dementia: Alzheimer's Cooperative Valuation in Europe recommendations. Int I Geriatr Psychiatry. 2014;29:682-93.

63. Lyle S, Grizzell M, Willmott S, Benbow S, Clark M, Jolley D. Treatment of a whole population sample of Alzheimer's disease with donepezil over a 4-year period: lessons learned. Dement Geriatr Cogn Disord. 2008;25:226-31.

64. Gustavsson A, Brinck P, Bergvall N, Kolasa K, Wimo A, Winblad B, et al. Predictors of costs of care in Alzheimer's disease: a multinational sample of 1222 patients. Alzheimers Dement. 2011;7:318-27.

65. Fitzpatrick-Lewis D, Warren R, Ali MU, Sherifali D, Raina P. Treatment for mild cognitive impairment: a systematic review and meta-analysis. CMAJ Open. 2015;3:E419-27.

66. Ritchie C, Smailagic N, Noel-Storr AH, Takwoingi Y, Flicker L, Mason SE, et al. Plasma and cerebrospinal fluid amyloid beta for the diagnosis of Alzheimer's disease dementia and other dementias in people with mild cognitive impairment (MCI). Cochrane Database Syst Rev. 2014;6:CD008782.

67. Santoro A, Siviero P, Minicuci N, Bellavista E, Mishto M, Olivieri F, et al. Effects of donepezil, galantamine and rivastigmine in 938 Italian patients with Alzheimer's disease. CNS Drugs. 2010;24:163-76.

68. Kuntz KM, Weinstein MC. Life expectancy biases in clinical decision modeling. Med Decis Making. 1995;15:158-69.

69. Consumer Reports. Evaluating prescription drugs used to treat Alzheimer's disease. 2012. http://www.consumerreports.org/cro/2012/07/evaluatingdrugs-to-treat-alzheimer-s-disease/index.htm. Accessed 16 July 2014

70. Payne G, Laporte A, Foot DK, Coyte PC. Temporal trends in the relative cost of dying: evidence from Canada. Health Policy. 2009;90:270-6.

\section{Submit your next manuscript to BioMed Central and we will help you at every step:}

- We accept pre-submission inquiries

- Our selector tool helps you to find the most relevant journal

- We provide round the clock customer support

- Convenient online submission

- Thorough peer review

- Inclusion in PubMed and all major indexing services

- Maximum visibility for your research

Submit your manuscript at www.biomedcentral.com/submit
Biomed Central 